\title{
Influence of SGLT2 Inhibitor Treatment on Urine Antioxidant Status in Type 2 Diabetic Patients: A Pilot Study
}

\author{
Diana Nabrdalik-Leśniak (D), ${ }^{1}$ Katarzyna Nabrdalik (D), ${ }^{1}$ Katarzyna Sedlaczek (iD, ${ }^{2}$

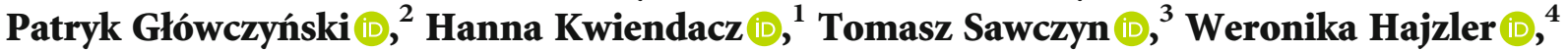 \\ Karolina Drożdż $\mathbb{D}^{1}{ }^{1}$ Mirela Hendel $\mathbb{D}^{2},{ }^{2}$ Krzysztof Irlik $\mathbb{D}^{2},{ }^{2}$ Pawel Stelmach $\mathbb{D}^{5},{ }^{5}$ \\ Piotr Adamczyk $\mathbb{D}^{\circ},{ }^{6}$ Andrzej Paradysz $\mathbb{D}^{5},{ }^{5}$ Slawomir Kasperczyk $\mathbb{D},{ }^{7}$ Tomasz Stompór $\mathbb{D}^{8},{ }^{8}$ \\ and Janusz Gumprecht $\mathbb{D}^{1}$ \\ ${ }^{1}$ Department of Internal Medicine, Diabetology and Nephrology in Zabrze, Faculty of Medical Sciences in Zabrze (41-800), \\ Medical University of Silesia, Katowice, Poland \\ ${ }^{2}$ Students' Scientific Association by the Department of Internal Medicine, Diabetology and Nephrology in Zabrze, Faculty of Medical \\ Sciences in Zabrze (41-800), Medical University of Silesia, Katowice, Poland \\ ${ }^{3}$ Department of Physiology in Zabrze, Faculty of Medical Sciences in Zabrze (41-800), Medical University of Silesia, Katowice, Poland \\ ${ }^{4}$ Doctoral School, Department of Pediatric Hematology and Oncology in Zabrze, Faculty of Medical Sciences in Zabrze (41-800), \\ Medical University of Silesia, Katowice, Poland \\ ${ }^{5}$ Department of Urology, Faculty of Medical Sciences in Zabrze (41-800), Medical University of Silesia, Katowice, Poland \\ ${ }^{6}$ Department of Pediatrics, Faculty of Medical Sciences in Katowice (40-752), Medical University of Silesia, Katowice, Poland \\ ${ }^{7}$ Department of Biochemistry, Faculty of Medical Sciences in Zabrze (41-800), Medical University of Silesia, Katowice, Poland \\ ${ }^{8}$ Department of Nephrology, Hypertension and Internal Medicine, University of Warmia and Mazury in Olsztyn, \\ Olsztyn (10-957), Poland
}

Correspondence should be addressed to Diana Nabrdalik-Leśniak; diananabrdalik@gmail.com

Received 1 February 2021; Revised 10 May 2021; Accepted 10 June 2021; Published 14 July 2021

Academic Editor: Ilaria Peluso

Copyright @ 2021 Diana Nabrdalik-Leśniak et al. This is an open access article distributed under the Creative Commons Attribution License, which permits unrestricted use, distribution, and reproduction in any medium, provided the original work is properly cited.

Sodium-glucose cotransporter 2 inhibitors (SGLT2i) have been recognized as potent antioxidant agents. Since SGLT2i are nephroprotective drugs, we aimed to examine the urine antioxidant status in patients with type 2 diabetes mellitus (T2DM). One hundred and one subjects participated in this study, including 37 T2DM patients treated with SGLT2i, 31 T2DM patients not using SGLT2i, and 33 healthy individuals serving as a control group. Total antioxidant capacity (TAC), superoxide dismutase (SOD), manganese superoxide dismutase (MnSOD), free thiol groups (R-SH, sulfhydryl groups), and catalase (CAT) activity, as well as glucose concentration, were assessed in the urine of all participants. Urine SOD and MnSOD activity were significantly higher among T2DM patients treated with SGLT2i than T2DM patients without SGLT2i treatment ( $p=0.009$ and $p=0.003$, respectively) and to the healthy controls $(p=0.002$ and $p=0.001$, respectively). TAC was significantly lower in patients with T2DM treated with SGLT2i when compared to those not treated and healthy subjects ( $p=0.036$ and $p=0.019$, respectively). It could be hypothesized that the mechanism by which SGLT2i provides nephroprotective effects involves improvement of the SOD antioxidant activity. However, lower TAC might impose higher OS (oxidative stress), and elevation of SOD activity might be a compensatory mechanism.

\section{Introduction}

Oxidative stress (OS) is defined as an imbalance between antioxidants and oxidants in favor of the latter, which leads to disruption of redox signaling and causes molecular damage [1]. Reactive oxygen species (ROS) constitute metabolites produced during physiological and metabolic processes and are neutralized by the antioxidant system [2]. OS occurs as 
a consequence of insufficient neutralization of ROS. It is well known that OS is involved in the development of diabetic vascular complications [2]. OS assessment can be based on measurement of ROS concentration, activities of antioxidant enzymes, and concentration of nonenzymatic antioxidative compounds [1]. Sodium-glucose cotransporter 2 inhibitors (SGLT2i), a new class of antidiabetic drugs, are thought to possess antioxidant properties. However, data on this issue come mostly from animal studies [3-6]. Inhibition of sodium-glucose cotransporter 2 (SGLT2) in proximal renal tubules increases urinary glucose excretion and decreases glycemia [7]. It is already known that besides the hypoglycemic effect, SGLT2i also provides cardiovascular benefits and nephroprotective effects through multiple biochemical pathways [8-13]. It has recently been revealed in large, randomized, placebo-controlled studies with canagliflozin [8-10], dapagliflozin [11], and empagliflozin [12,13] that these drugs, in addition to their already proven cardioprotective properties, also exert substantial nephroprotective effects. These effects seem to be independent of glucoselowering efficacy, but the exact mechanism of this action remains to be clarified. One of the hypotheses of these protective cardiorenal effects may be the potential for OS reduction preventing the free-radical generation [3-5] and enhanced antioxidant defense by increased activity of protective enzymes [14]. Unfortunately, studies assessing this phenomenon in patients treated with SGLT2i are scarce.

Superoxide is the primary ROS that links hyperglycemia and pathways engaged in developing vascular complications of T2DM. In turn, superoxide dismutase (SOD) is important for each cell because it leads to superoxide scavenging, and its isoform, manganese superoxide dismutase (MnSOD) localized in mitochondria, is considered the first-line defense against ROS [15]. Other important protective mechanisms include catalase (CAT) [16] and free thiol groups (R-SH, sulfhydryl groups) [17]. Activities of the enzymes mentioned above and concentrations of nonenzymatic factors can be measured to assess the redox status and intensiveness of an OS. It is also possible to measure total antioxidant capacity (TAC), which delivers information related to overall radical removal ability [18]. To date, most of the studies that demonstrate OS reduction caused by SGLT2 inhibition have been conducted on animal models with the use of blood and tissues of the studied animals [3-6]. There have also been attempts to examine OS in urine, which is tempting since it is a noninvasive, inexpensive, and simple to conduct assessment [19-24]. In the presented study, we hypothesized that treatment with SGLT2i would impact urinary antioxidant enzymes and nonenzymatic biomolecules as indicators of the antioxidant barrier. Urine examination was chosen because of its efficiency, material collection simplicity, and the possibility of using this method in further studies on a larger group of patients.

\section{Materials and Methods}

2.1. Subjects. This was an observational study of patients treated in the Outpatient Diabetology Clinic in the University Hospital in Zabrze, Poland. The consecutive eligible patients who fulfilled the inclusion criteria were invited to participate in the study. There were three groups of study participants: patients with T2DM treated with SGLT2i (group no 2), diabetic controls (i.e., patients with T2DM not treated with SGLT2i) (group no 1), and healthy controls (group no 3). Consecutive patients coming for a routine visit in the outpatient diabetology clinic who fulfilled the inclusio$\mathrm{n}$ /exclusion criteria based on the medical documentation and interview were assigned into the groups. Healthy controls were recruited from the medical staff of the Hospital where the Outpatient Diabetology Clinic is located. The information about the recruitment process could be found on leaflets located in the Outpatient Diabetology Clinic.

The inclusion criteria for the study groups were age $\geq$ 18 years, T2DM of at least of 12 months duration, and treatment with SGLT2i for at least one month. The inclusion criteria for the diabetic controls were age $\geq 18$ years, T2DM of at least of 12 months duration, and treatment with any antidiabetic drug except SGLT2i.

The exclusion criteria for the study groups were eGFR (estimated glomerular filtration rate) $<60 \mathrm{ml} / \mathrm{min} / 1.73$ $\mathrm{m}^{2}$, clinical signs of urinary tract infection and any other ongoing acute illness since these conditions could directly affect urine OS. The inclusion criteria for the control group were as follows: age $\geq 18$ years and participants who had recent (assessed within less than three months) serum creatinine concentration within the reference range (assessed as a routine screening method performed in medical staff). The exclusion criteria for the control group was as follows: any chronic or acute illness at the time of enrolment into the study.

The medical history, including data regarding diabetes duration, demographic characteristics, comorbidities, and concomitant medications, was obtained from the medical records. $\mathrm{HbA}_{1 \mathrm{c}}$ (glycated hemoglobin $\mathrm{A}_{1 \mathrm{c}}$ ) was assessed as a routine part of outpatient diabetes management. Body mass and height were measured by standard methods. BMI (body mass index) was calculated among all participants, and the first morning urine void was collected on the day of the visit to the Outpatient Diabetology Clinic. Healthy volunteers had body mass and height measured by standard methods and BMI calculated, and first morning urine void collected on the day of informed consent was signed (leaflets advertising the study contained instructions on how to collect the firstmorning urine void). The study protocol was exempt from the necessity of obtaining the approval of the Ethics Committee by the Medical University of Silesia due to its observational nature (KNW/0022/KB/33/19). Informed consent was obtained from all participants before inclusion into the study.

2.2. Biochemical Analysis-Blood Samples. Venous blood samples were taken as a routine part of diabetes management for evaluation of plasma $\mathrm{HbA}_{1 \mathrm{c}}$ and serum creatinine concentration with subsequent eGFR calculation during the patient's visit to the outpatient diabetology clinic. $\mathrm{HbA}_{1 \mathrm{c}}$ was measured with the HLPC (high-performance liquid chromatography) method [25], creatinine was measured with Jaffe's colorimetric assay [26] (Cobas Integra 800, Roche Diagnostics $\left.{ }^{\circledR}\right)$, and eGFR was calculated according to CKDEPI formula [27]. 
2.3. Biochemical Analysis-Urine Samples. The urine samples were collected from the fresh urine, the first void of the day into $75 \mathrm{ml}$ sterile containers. All urine specimens were stored frozen at $-80^{\circ} \mathrm{C}$ until testing at the end of the collection period. All urine samples were analyzed for glucose, creatinine with subsequent urinary albumin-to-creatinine ratio (UACR) calculation as well as SOD, MnSOD, TAC, R-SH, and CAT.

2.3.1. Urine Glucose Concentration. Urine glucose concentration was measured by the colorimetric enzymatic method (Alpha Diagnostics ${ }^{\circledR}$ test) [28]. The glucose oxidase enzyme catalyzes the oxidation of glucose to gluconic acid and hydrogen peroxide [29]. The hydrogen peroxide oxidizes an oxygen acceptor to give chromogenic oxidation products; the intensity of its color is proportional to the amount of glucose [29]. Glucose concentration was expressed as milligram per decilitre of urine $(\mathrm{mg} / \mathrm{dl})$.

2.3.2. UACR Measurement. Urine albumin concentration was measured with the turbidimetric immunoassay [30] (Cobas c501, Roche Diagnostics ${ }^{\circledR}$ ), and urine creatinine concentration was determined by the Jaffe colorimetric assay [26]. UACR ( $\mathrm{mg} / \mathrm{g}$ creatinine) that has been calculated as albumin concentration $(\mathrm{mg} / \mathrm{L})$ divided by creatinine concentration $(\mathrm{g} / \mathrm{L})$ according to guidelines $[30,31]$.

2.3.3. Urine Total SOD and MnSOD Activity. Urine total SOD activity, which is constituted by activities of all SOD isozymes, that is, extracellular SOD and both intracellular isoforms CuZnSOD and MnSOD, was measured according to the Oyanagui method $[32,33]$. Superoxide anion, with the participation of xanthine oxidase, reacts with hydroxylamine creating nitrate ions, which after connection with naftylenodiamin and sulfanilic acid, produces color; for MnSOD measurement, potassium cyanide was used to deactivate other enzymes, including CuZnSOD and extracellular SOD $[32,33]$. Reading was calculated against a blank probe consisted of bidestilled water, measured at a wavelength of 560 nm with Victor X3 Perkin Elmer ${ }^{\circledR}$ reader (Waltham, Mass., USA). This method is completely specific for SOD. The enzyme activity was expressed as nitrite units (NU) per $\mathrm{ml}$ of urine $(\mathrm{NU} / \mathrm{ml})$. One NU means the ability to $50 \%$ reduction of nitrate ion production in the presence of SOD.

2.3.4. Urine TAC. Urine $T A C$ was measured according to the Erel method. First, ABTS $\left(2,2^{\prime}\right.$-azinobis(3-ethylbenzothiazoline-6-sulfonic acid)) is oxidized to ABTS. ${ }^{+}$radical cation by hydrogen peroxide [34]. Spontaneous reduction of ABTS. ${ }^{+}$ and thus, decolorization of its green solution is then accelerated by antioxidants, with a certain rate, depending on their concentrations and antioxidant capacities [34]. Color change was measured as a change of absorbance at a wavelength of $650 \mathrm{~nm}$ on the Victor X3 Perkin Elmer ${ }^{\circledR}$ reader. The result was determined from the standard curve, and an assay was calibrated by Trolox. TAC was expressed as $\mathrm{mmol} / \mathrm{l}$ urine.

2.3.5. Urine Free Thiol Groups. Urine free thiol group (R-SH, sulfhydryl groups) concentration was measured according to the Koster modified half-automatic method using the Victor
X3 Perkin Elmer ${ }^{\circledR}$ reader at a wavelength of $405 \mathrm{~nm}$ [35]. This method is based on the reduction of DTNB $\left(5,5^{\prime}\right.$ -ditiobis(2-nitrobensoid) acid) by chemical compounds containing sulfhydryl groups, and as a result, the yellow 5-tio-2nitrobensoid anion is produced [35]. R-SH concentration was calculated from the standard curve; the sample was calibrated with glutathione. R-SH concentration was expressed as $\mu \mathrm{mol} / \mathrm{l}$ urine.

2.3.6. Urine CAT Activity. CAT activity was measured according to the peroxidase method with Purpald (4amino-3-hydrazino-5-mercapto-1,2,4-triazole) as chromogen [36]. This method is based on the reaction of the enzyme with methanol in the presence of an optimal concentration of hydrogen peroxide [36]. Produced formaldehyde was measured with Victor X3 Perkin Elmer ${ }^{\circledR}$ reader at the wavelength of $560 \mathrm{~nm}$. Calibration was performed with formaldehyde. CAT activity was expressed as IU/l urine.

2.4. Statistical Analysis. The descriptive statistics of continuous variables were expressed as median (min-max) and 95\% confidence interval (CI). The Shapiro-Wilk test was used to verify the normality of data distribution. For normally distributed variables, the independent Student $t$-test was applied for comparisons between groups. The $\chi^{2}$ Pearson's test was used for comparison between groups for categorized variables. The homogeneity of variance was checked using Levene's test. When more than two subgroups were compared, analysis of variance (ANOVA) and posthoc verification with the least significant difference test (LSD) and Bonferroni correction was utilized. Pearson's correlation coefficient measured the strength of the association between two variables. $p$ value $<0.05$ was considered significant. Statistical analysis was conducted using Statistica Statsoft ${ }^{\circledR}$ version 13.3.

\section{Results}

3.1. Population Characteristics. The study included 101 participants. The demographic and clinical characteristics of the study subjects are described in Table 1 . There were 37 patients (group 2) with T2DM treated with SGLT2i for at least 1 month (empagliflozin, $n=19$ and canagliflozin, $n=$ 18) and a control group of 31 patients (group 1) with T2DM not treated with SGLT2i and 33 healthy individuals (group 3). There were significantly fewer men in the control group of healthy individuals than the patients treated $(p=0.012)$ or not treated $(p=0.0055)$ with SGLT2i. There was also a significant difference in age between the studied groups of patients and the healthy control group ( $p=0.0012$ for comparison of T2DM patients treated with SGLT2i and healthy controls and $p=0.0001$ in case of comparing T2DM not treated with SGLT2i to healthy controls). There was also a significant difference in diabetes duration between patients treated or not with SGLT2i $(p=0.032)$. The only comorbidity among patients with diabetes was hypertension, and patients treated and not treated with SGLT2i did not differ in terms of the prevalence of hypertension, as well as the number and class of blood pressure- 
Table 1: Demographic and clinical characteristics of the study subjects.

\begin{tabular}{|c|c|c|c|c|}
\hline Variables & $\begin{array}{l}\text { T2DM patients not treated with } \\
\text { SGLT2is (1) } n=31\end{array}$ & $\begin{array}{l}\text { T2DM patients treated with } \\
\text { SGLT2is (2) } n=37\end{array}$ & $\begin{array}{l}\text { Healthy controls (3) } \\
n=33\end{array}$ & $p$ value \\
\hline Male $[n(\%$ men $)]$ & $17(55)$ & $22(59)$ & $7(21)$ & $<0.01^{*}$ \\
\hline Mean age [years] & $\begin{array}{c}60.0(42.0-80.0 \\
95 \% \text { CI: } 56.7-62.7)\end{array}$ & $\begin{array}{c}65.0 \text { (39.0-78.0; } \\
95 \% \text { CI: 60.4-66.1) }\end{array}$ & $\begin{array}{c}51.0(40.0-63.0 \\
95 \% \text { CI: 49.3-53.2) }\end{array}$ & $<0.001^{* *}$ \\
\hline Diabetes duration [years] & $\begin{array}{c}6.0 \text { (0.00-20.00; } \\
95 \% \text { CI: } 5.8-10.2)\end{array}$ & $\begin{array}{c}13.0(3.0-23.0 \\
95 \% \text { CI: } 27.8-31.1)\end{array}$ & - & $<0.01^{* * *}$ \\
\hline $\mathrm{HbA}_{1 \mathrm{c}}[\%]$ & $\begin{array}{c}7.9(6.2-11.4 \\
95 \% \mathrm{CI} ; 7.6-8.6)\end{array}$ & $\begin{array}{c}7.6 \text { (6.4-10.0; } \\
95 \% \text { CI: } 7.3-7.9)\end{array}$ & - & $>0.05^{* * *}$ \\
\hline BMI $[\mathrm{kg} / \mathrm{m} 2]$ & $\begin{array}{c}29.7(22.8-42.5 \\
95 \% \text { CI: } 28.5-31.6)\end{array}$ & $\begin{array}{c}28.7(21.9-52.7 \\
95 \% \text { CI: } 27.8-31.3)\end{array}$ & $\begin{array}{c}26.6(21.6-30.1 \\
95 \% \text { CI: } 25.4-27.0)\end{array}$ & $<0.001^{* *}$ \\
\hline $\mathrm{eGFR}\left[\mathrm{ml} / \mathrm{min} / 1,73 \mathrm{~m}^{2}\right]$ & $\begin{array}{c}90.0 \text { (62.3-106.1; } \\
95 \% \text { CI: 82.3-91.0) }\end{array}$ & $\begin{array}{c}\text { 92.5(62.1.3-113.7; } \\
95 \% \text { CI: 82.8.-92.6) }\end{array}$ & $\begin{array}{l}\text { 91,n(71.6-115.7; } \\
\text { 95\% CI: 85.8-93.7) }\end{array}$ & $>0.05^{* *}$ \\
\hline $\begin{array}{l}\text { Urinary albumin } \\
\text { concentration }[\mathrm{mg} / \mathrm{l}]\end{array}$ & $\begin{array}{c}6.3 \text { (0.2-16.5; } \\
95 \% \text { CI: } 4.9-8.3)\end{array}$ & $\begin{array}{c}4.2(0.8-13.4 ; \\
95 \% \text { CI: } 3.8-6.2)\end{array}$ & $\begin{array}{c}4.3 \text { (1.2-9.4; } \\
95 \% \text { CI: } 4.0-5.4)\end{array}$ & $>0.05^{* * *}$ \\
\hline $\begin{array}{l}\text { Urinary creatinine } \\
\text { concentration }[\mathrm{g} / \mathrm{l}]\end{array}$ & $\begin{array}{c}0.5(0.1-0.7 \\
95 \% \text { CI: } 0.4-0.5)\end{array}$ & $\begin{array}{c}0.4(0.1-0.7 \\
95 \% \text { CI: } 0.4-0.5)\end{array}$ & $\begin{array}{c}0.4(0.2-0.7 \\
95 \% \text { CI: } 0.4-0.5)\end{array}$ & $>0.05^{* * *}$ \\
\hline UACR $[\mathrm{mg} / \mathrm{g}]$ & $\begin{array}{c}13.3(0.7-25.1 \\
95 \% \text { CI: } 10.6-15.3)\end{array}$ & $\begin{array}{c}11.8(1.8-21.8 ; \\
95 \% \text { CI: 9.4-12.9) }\end{array}$ & $\begin{array}{c}10.4(2.1-15.1 \\
95 \% \text { CI: 9.3-11.4) }\end{array}$ & $>0.05^{* * *}$ \\
\hline ACEI or ARB $[n(\%)]$ & $8(25.8)$ & $10(27.0)$ & $0(0)$ & $>0.05^{*}$ \\
\hline Beta-blockers $[n(\%)]$ & $4(12.9)$ & $5(13.5)$ & $0(0)$ & $>0.05^{*}$ \\
\hline Hypertension $[n(\%)]$ & $10(32.0)$ & $11(30.0)$ & $0(0)$ & $>0.05^{*}$ \\
\hline
\end{tabular}

lowering medications. Healthy volunteers were not using any drugs in the long-term.

ACEI: angiotensin-converting enzyme inhibitors; ARB: angiotensin II receptor blockers; BMI: body mass index; eGFR: estimated glomerular filtration rate; $\mathrm{HbA}_{1 \mathrm{c}}$ : glycated hemoglobin $\mathrm{A}_{1 \mathrm{c}}$; T2DM: type 2 diabetes mellitus; SGLT2i: sodium-glucose cotransporter 2 inhibitor; UACR: urine albumin-to-creatinine ratio; $\mathrm{n}$ : number of patients; $p$ value: level of significance; ${ }^{*} \chi^{2},{ }^{* *}$ ANOVA, ${ }^{* * *}$ student $t$-test. Continuous variables are presented as median (min-max; $95 \%$ CI).

3.2. Antioxidant Status. Table 2 displays the comparison of urine antioxidant status and urine glucose concentration between both T2DM patient groups and healthy controls. Presented differences are independent of patients' age, which was proved in the ANOVA analysis.

T2DM: type 2 diabetes mellitus; SGLT2i: sodium-glucose cotransporter 2 inhibitors; SOD: superoxide dismutase; MnSOD: manganese superoxide dismutase; TAC: total antioxidant capacity; R-SH: sulfhydryl groups; CAT: catalase; n: number of patients. Continuous variables are presented as median (min-max; 95\% CI).

LSD tests' significance: ${ }^{a} p<0.01$ (between (1) and (2)), ${ }^{\mathrm{b}} p<0.001$ (between (2) and (3)), ${ }^{\mathrm{c}} p<0.01$ (between (1) and (2)), ${ }^{\mathrm{d}} p<0.001$ (between (2) and (3)), ${ }^{\mathrm{e}} p<0.01$ (between (1) and (2)), ${ }^{f} p<0.05$ (between (2) and (3)), ${ }^{\mathrm{g}} p<0.05$ (between (1) and (3)), ${ }^{\mathrm{h}} p<0.01$ (between (2) and (3)), ${ }^{\mathrm{i}} p<0.001$ (between (1) and (2)), ${ }^{\mathrm{j}} p<0.001$ (between (1) and (3)), and ${ }^{\mathrm{k}} p<0.001$ (between (2) and (3)).

We also performed the analysis of the possible relationship between glucosuria and the activities of the antioxidant enzymes. Analysis revealed significant positive correlations with CAT, SOD, and MnSOD (Figures 1, 2, and 3, respectively). SOD and MnSOD positive correlation stay in line with a significantly higher level of these enzymes in patients treated with SGLT2i.

\section{Discussion}

In this study, we report that the urine antioxidant status in patients treated with SGLT2i differs significantly from the one measured in T2DM patients not treated with SGLT2i, as well as from healthy controls. To date, only a limited number of studies have attempted to assess antioxidant enzyme activities in the urine of patients with T2DM, and neither one was performed in patients treated with SGLT2i. Hence, the direct comparison of our findings with other studies is not possible. Therefore, this discussion is focused on antioxidant status in blood and tissues in studies performed on animal models. In the presented study, among measured antioxidative enzymes, only CAT activity was comparable between the studied groups, whereas urine SOD and MnSOD activity was significantly higher among T2DM patients treated with SGLT2i compared to diabetic controls not receiving SGLT2 $\mathrm{i}$ and to healthy controls. TAC was significantly lower in patients with T2DM treated with SGLT2i when compared to those untreated with SGLT2i and healthy subjects. In the case of R-SH, its concentration was significantly lower in patients with T2DM treated with SGLT2i than healthy controls. Yet, among patients with T2DM treated with SGLT2i and treated otherwise, the difference was not statistically significant. 
TABLE 2: Comparison of antioxidant status and glucose concentration in urine among studied groups.

\begin{tabular}{|c|c|c|c|c|}
\hline Variables & $\begin{array}{l}\text { T2DM patients not treated with } \\
\text { SGLT2i (1), } n=31\end{array}$ & $\begin{array}{l}\text { T2DM patients treated with } \\
\text { SGLT2i }(2), n=37\end{array}$ & $\begin{array}{l}\text { Healthy controls (3), } \\
\qquad n=33\end{array}$ & $p$ ANOVA \\
\hline Total SOD [NU/ml] & $\begin{array}{c}25.2(8.9-35.8 \\
95 \% \text { CI: } 21.1-26.2)^{a}\end{array}$ & $\begin{array}{c}28.5(16.7-36.0 \\
95 \% \text { CI: } 26.1-29.4)^{\mathrm{a}, \mathrm{b}}\end{array}$ & $\begin{array}{c}23.2(9.7-25.1 ; \\
95 \% \text { CI: } 20.2-25.2)^{b}\end{array}$ & $<0.01$ \\
\hline $\mathrm{MnSOD}[\mathrm{NU} / \mathrm{ml}]$ & $\begin{array}{c}22.6(8.6-35.9 ; \\
95 \% \text { CI: } 19.1-24.4)^{c}\end{array}$ & $\begin{array}{c}27.1(14.3-35.9 ; \\
95 \% \text { CI: } 24.9-28.5)^{\mathrm{c}, \mathrm{d}}\end{array}$ & $\begin{array}{c}21.6(8.34-35.1 ; \\
95 \% \text { CI: } 18.8-23.9)^{\mathrm{d}}\end{array}$ & $<0.001$ \\
\hline $\mathrm{TAC}[\mathrm{mmol} / \mathrm{l}]$ & $\begin{array}{c}9.9(0.0-45.8 ; \\
95 \% \text { CI: } 7.9-14.6)^{\mathrm{e}}\end{array}$ & $\begin{array}{c}7.9(0.0-29.1 ; \\
95 \% \text { CI: } 6.5-11.7)^{\mathrm{e}, \mathrm{f}}\end{array}$ & $\begin{array}{c}9.2(0.4-51.2 ; \\
95 \% \text { CI: 8.4-15.2) }\end{array}$ & $<0.05$ \\
\hline $\mathrm{R}-\mathrm{SH}[\mu \mathrm{mol} / \mathrm{l}]$ & $\begin{array}{c}1.7(0.0-51.0 ; \\
95 \% \text { CI: } 4.5-16.2)^{\mathrm{g}}\end{array}$ & $\begin{array}{c}1.9(0.0-54.7 \\
95 \% \text { CI: } 3.4-11.8)^{\mathrm{h}}\end{array}$ & $\begin{array}{c}13.6(0.0-117.9 ; \\
95 \% \text { CI: } 10.8-30.6)^{\mathrm{g}, \mathrm{h}}\end{array}$ & $<0.05$ \\
\hline CAT [IU/l] & $\begin{array}{c}2.9 \text { (1.0-8.4; } \\
95 \% \text { CI: } 2.5-3.6)\end{array}$ & $\begin{array}{c}3.4(1.3-16.4 \\
95 \% \text { CI: } 3.2-4.9)\end{array}$ & $\begin{array}{c}2.6(0.6-9.5 \\
95 \% \text { CI: } 2.3-3.6)\end{array}$ & $>0.05$ \\
\hline $\begin{array}{l}\text { Urinary glucose } \\
\text { concentration [mg/dl] }\end{array}$ & $\begin{array}{c}305(49.0-3346.3 ; \\
95 \% \text { CI: 269.9-897.7) }\end{array}$ & $\begin{array}{c}2699.3(85.7-3447.7 ; \\
\text { 95\% CI: } 2190.9-2742.0)^{\mathrm{i}, \mathrm{k}}\end{array}$ & $\begin{array}{c}2.8(0.0-9.4 \\
95 \% \text { CI: } 2.0-3.7)^{j, k}\end{array}$ & $<0.001$ \\
\hline
\end{tabular}

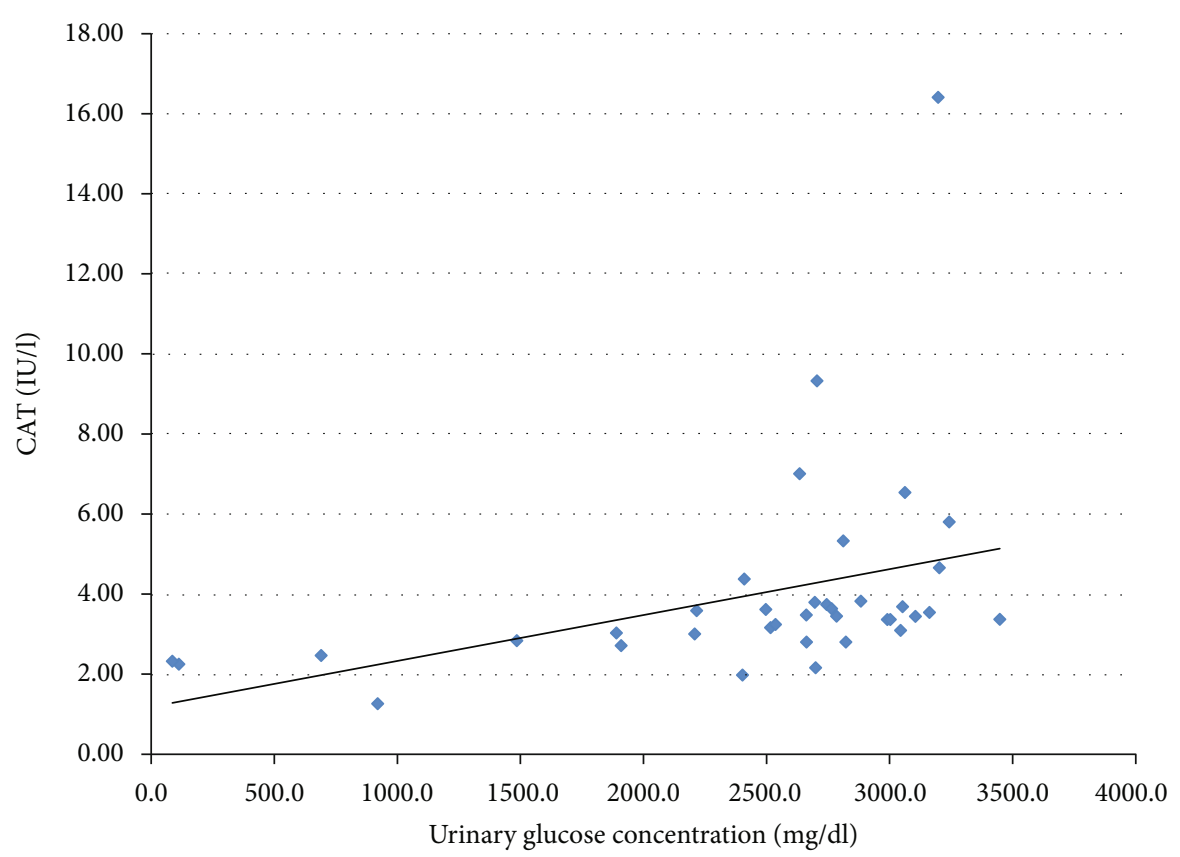

FIGURE 1: Correlation between urine CAT activity and urine glucose concentration in a group of patients treated with SGLT2i $(p<0.001)$.

On the other hand, R-SH concentration was significantly lower in patients with T2DM not using SGLT2i than healthy individuals. This observation might suggest that $\mathrm{R}-\mathrm{SH}$ concentration is related rather to diabetes itself than to the mode of antidiabetic treatment. Yet, it needs further studies in larger groups of patients.

Since the results stemming from the animal studies implicate that SGLT2i mode of action is to reduce the OS [3-6], in this context, higher activity of the mentioned enzymes is surprising because this suggests that the OS is actually higher under this treatment. Previous studies performed in patients with diabetes revealed that TAC was lower among patients with chronic renal failure on maintenance dialysis [37], and total R-SH group concentration in urine of patients with diabetic kidney disease was similar to healthy subjects (although its serum concentration was lower in patients with diabetes) [20]. Similarly, other studies indicate that decreased systemic R-SH groups directly reflect increased whole body OS [38-41]. On the other hand, Shin et al., in their studies on animal models, demonstrated that dapagliflozin reduced OS by increasing MnSOD, Cu/ZnSOD, and catalase expression in renal tissues of animals with diabetic kidney disease [5]. As mentioned in the introduction, in addition to an already known protective effect in cardiovascular diseases, SGLT2i were recently found to have nephroprotective properties. ACEI (angiotensin-converting enzyme inhibitors), another class of nephroprotective agents, also contributes to protection against oxidative stress, for example, leading to the enhancement of SOD activity [42]. Therefore, one might hypothesize that for SGLT2i, the case might be similar. However, it must be kept in mind that this association was observed in blood, not in urine.

Also, the data on antioxidant enzyme activities in diabetes is controversial, and studies present equivocal results. 


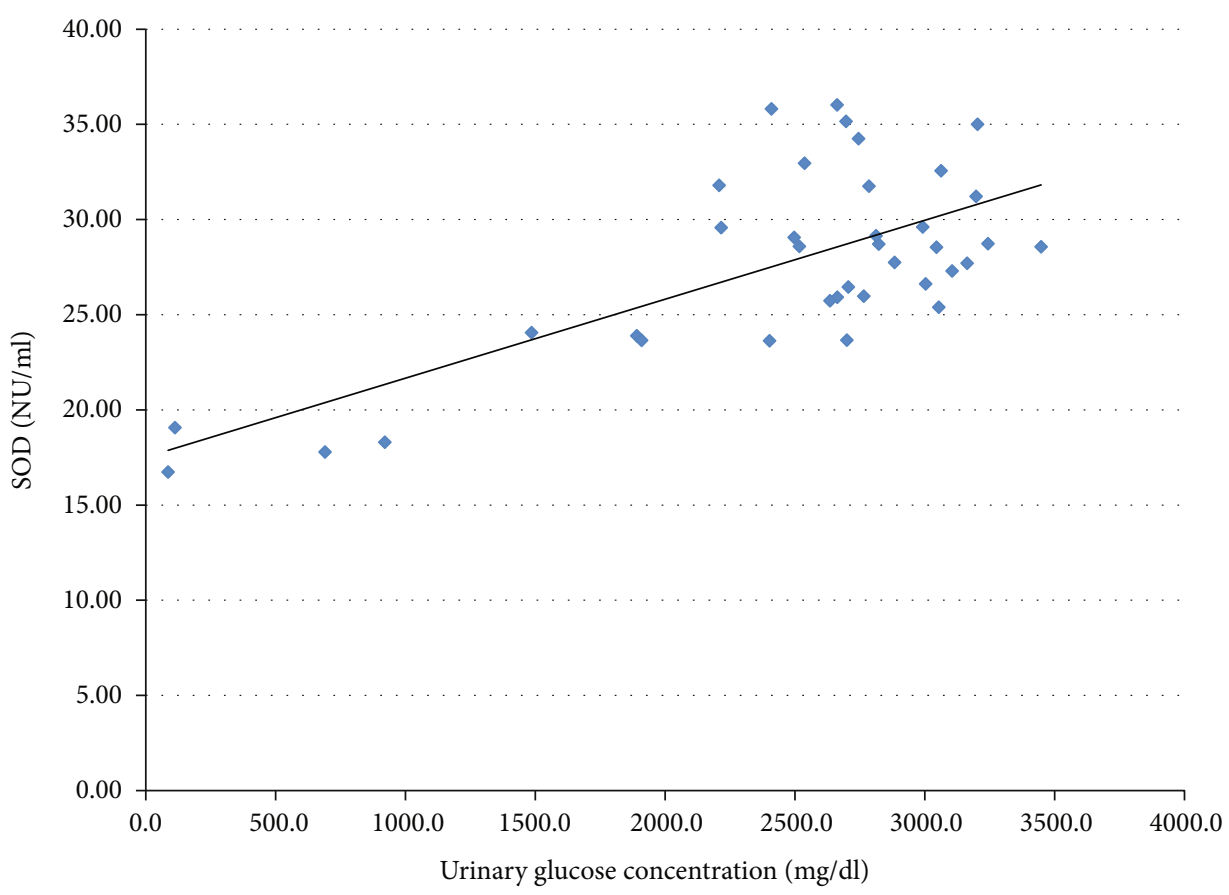

FIGURE 2: Correlation between urine SOD activity and urine glucose concentration in a group of patients treated with SGLT2i $(p<0.001)$.

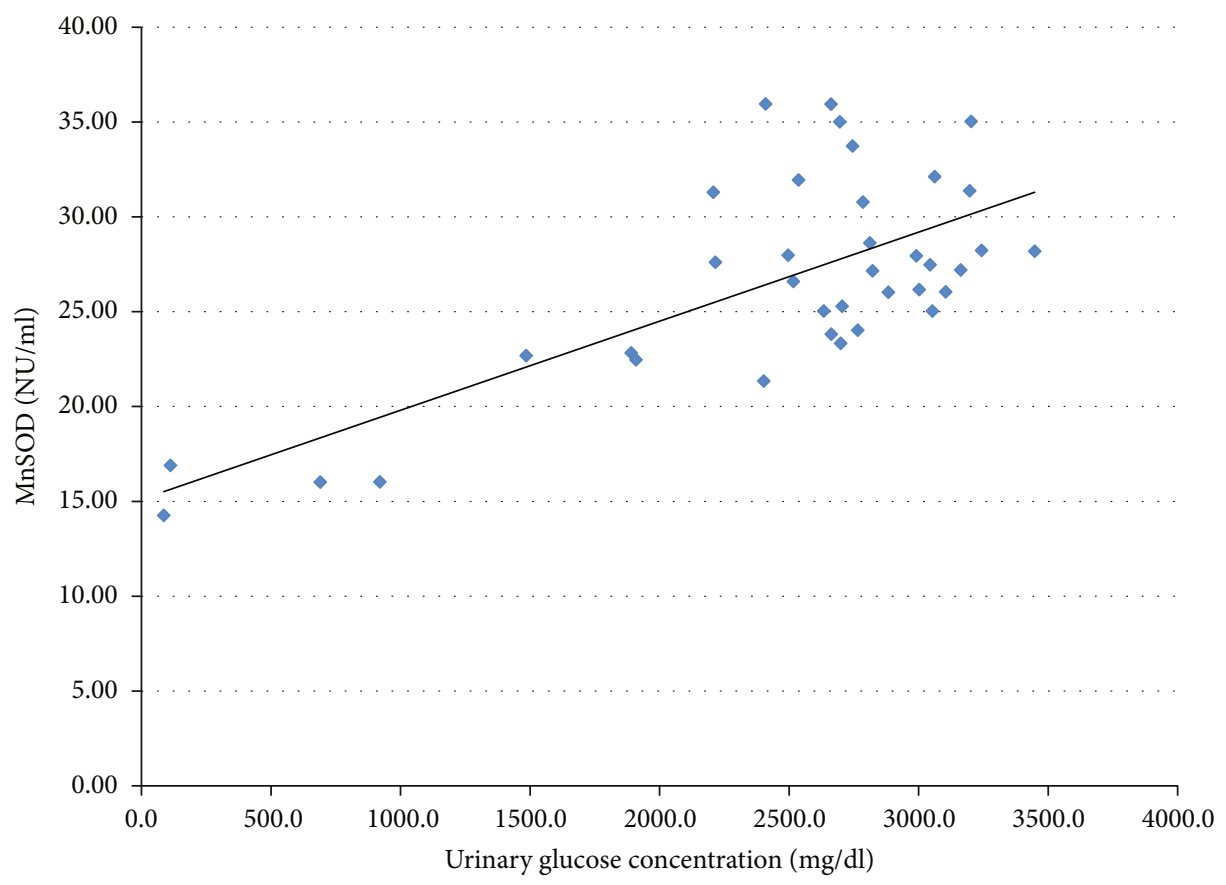

FIGURE 3: Correlation between urine MnSOD activity and urine glucose concentration in a group of patients treated with SGLT2i ( $p<0.001)$.

Sundaram et al. [43] revealed in their experiment that plasma and erythrocyte SOD and CAT activities were decreased in patients with diabetes when compared to the nondiabetics. Similarly, Hartnett et al. [44] found reduced SOD activity in patients with diabetes. They suggested that increased activity of SOD might serve as a compensatory mechanism what was also mentioned as a reason for the elevation of plasma SOD activity in patients with T2DM in the study by Turk et al. [45].
While focusing on urine antioxidant activity studied to date in patients with diabetes, it is worth mentioning the study performed by Gul et al., who measured urinary antioxidant enzyme activities in T2DM subjects with urinary tract infection compared them with healthy subjects [46]. These authors revealed that both SOD and CAT activities were significantly lower in T2DM subjects with urinary tract infection than healthy controls, suggesting that elevated OS caused enzyme consumption [46]. Liu et al. demonstrated a 
higher concentration of OS biomarkers in the urine of T2DM patients with vascular complications as compared to those without it [24].

Additionally, since SGLT2i treatment leads to glucosuria, we performed a correlation analysis of glucosuria and antioxidant enzyme activity, which revealed significant positive correlations with respect to CAT, SOD, and MnSOD. These positive correlations are consistent with significantly higher SOD and MnSOD activity among patients treated with SGLT2i, whereas CAT activity seemed not to be affected by this treatment. Because no information regarding glucosuria and OS could be found in the literature, we can only hypothesize that glucosuria might stimulate OS and antioxidative defense by increasing SOD and MnSOD activity. Proximal tubules under treatment with SGLT2i are largely protected from glucose toxicity since their uptake by these cells is minimized. At the same time, tubular filtrate that reaches more distally located nephron segments is significantly enriched with glucose. Most of the recent interest concerning glucose handling by the kidney has been focused on the glucose uptake in the proximal nephron and the role of SGLT1 and SGLT2. Still, absorption of glucose in the loop of Henle and distal nephron segments (although much less important from a physiologic point of view) has also been confirmed $[47,48]$. The apical glucose transporter (GLUT) transporters have been identified in the apical membranes of late nephron segment cells and were shown to be upregulated in the experimental models of diabetes; renal GLUT knockout can also induce glycosuria [49]. In the available literature, we did not find any data on glucose handling by the distal nephron following treatment with SGLT2i (although it has been shown that increased reabsorption of glucose by GLUT9 in proximal tubule following SGLT2i treatment is one of the mechanisms that competitively lowers uric acid reabsorption in this part of nephron) [50]. We hypothesize that increased glucose influx into the cells of distal nephron during the treatment with SGLT2i might inhibit Nrf2 (nuclear factor erythroid 2-related factor 2, the master transcription factor controlling defense against OS), thus enhancing OS in this part of nephron $[51,52]$. It cannot be ruled out that to counterbalance this effect, certain antioxidant systems (possibly not controlled by Nrf2) might become enhanced in this part of nephron and result in increased activity of enzymes with an antioxidative potential in the final urine (as observed in our study). Several drugs which already used to treat diabetes or under the investigation as potential therapeutic agents, such as metformin, glucagon-like peptide-1 receptor (GLP1R) agonists, and bardoxolone, were shown to upregulate Nrf2 [53, 54]; we did not find such data for SGLT2i except for limited data on an animal model where empagliflozin is thought to promote the nuclear translocation of Nrf2 and limit the OS in the heart of mice with diabetes [3]. In our opinion, it is tempting to test such a hypothesis in an experimental study focusing on glucose handling by the distal nephron following the treatment with SGLT2i. Recently, redox balance has been characterized for the first time in saliva and blood of participants in different age groups revealing that antioxidant barrier decreases with age [55].Taking this information into account, an interesting direction of future research in this field would be inclusion of patients differing in age, especially elderly ones, since the human population is aging, and this favors diabetes complications occurrence.

In addition to OS, investigating the advanced end glycation products (AGEs) also seems a promising direction for future studies. AGEs are generated upon the nonenzymatic reactions between glucose and other sugars with proteins, lipids, or nucleic acids and participate in the pathogenesis of diabetic vascular complications [56-59]. AGEs can be related to oxidative stress because their formation starts under hyperglycaemic and/or oxidative stress conditions [60]. On the other hand, inhibiting the glycation process may be the way to limit the diabetes related complications [61]. Since there has been albumin glycation inhibition by metformin and glipizide demonstrated, in vitro study [62] maybe also SGLT2i may present this mode of action. The study performed most recently with another nephroprotective class of drugs-angiotensin II receptor blockers (ARB) - demonstrated their antiglycooxidant activity. These findings support the need for future studies in this area with the use of SGLT2i [63]. Given the fact that some literature reports suggest SGLT2i interact with AGEs during their formation, the next step in future studies could address this matter further [64-66]. For example, administration of empagliflozin for 4 weeks improved hyperglycemia and lowered $\mathrm{HbA}_{1 \mathrm{c}}$ level and resulted in a decreased expression of AGEs in kidneys of rats with streptozocin-induced diabetes [64]. Dapagliflozin ameliorated glucotoxicity in human renal proximal tubular epithelial cells by preventing AGE formation and synthesis of the proinflammatory cytokines such as transforming growth factor beta 1 (TGF- $\beta 1$ ) and interleukin 8 (IL-8) [65]. A potential of AGEs to induce apoptosis of tubular epithelial cells through and interaction with AGEreceptor (RAGE) was ameliorated by the SGLT2 inhibition in a study conducted on cultured human renal proximal tubular epithelial cells [66]. Limitation of studies concerning the impact of SGLT2i on AGEs conducted on human cells suggests the need for further investigation in this field, with the inclusion of T2DM patients treated with SGLT2i.

\section{The Limitations of the Study}

The limitations of the study include differences in demographic characteristics of the examined groups (as there were more women among healthy individuals and those participants were younger than patients with T2DM) and differences in age and duration of T2DM between patients treated or not treated with SGLT2i. However, taking into account the mechanism of action of SGLT2i and its independence from endogenous insulin secretion, duration of diabetes should not affect the obtained results. Even though these limitations are acknowledged shortcomings of observational studies, it has been demonstrated recently that gender does not influence level of oxidative stress [55] but one must keep in mind that age differences could influence presented outcomes. It should also be noted that due to the small size of the group using ARBs, no additional analysis of their potential impact on the parameters of oxidative stress was 
performed. Given the fact that some recent literature reports suggest SGLT2i interact with AGEs during their formation and consequently affect the intensity of oxidative stress, the next step in future studies could be addressing this matter further.

\section{Conclusions}

In the presented study, we have demonstrated that treatment with SGLT2i influences urine antioxidant status in patients with type 2 diabetes. It could be hypothesized that the outcomes of the presented study indicate that the mechanism by which SGLT2i provides nephroprotective effects involves improvement of the SOD antioxidant defense. It would be of great value to elucidate the mechanism underlying the upregulation of SOD induced by SGLT2i in future studies. On the other hand, lower TAC might impose higher OS in the urine of patients treated with SGLT2i, and elevation of SOD activity might be a compensatory mechanism. Further studies are needed to replicate these results and resolve why TAC was lowered among patients treated with SGLT2i. It would also be interesting to assess oxidative urine status in prospective serial measurements in the same group of patients.

\section{Data Availability}

The data presented in this study are available on request from the corresponding author. The data are not publicly available due to privacy.

\section{Ethical Approval}

Ethical review and approval were waived for this study due to its observational nature (KNW/0022/KB/33/19).

\section{Consent}

An informed consent was obtained from all participants before the inclusion into the study.

\section{Conflicts of Interest}

The authors declare no conflict of interest.

\section{Authors' Contributions}

All authors have read and agreed to the published version of the manuscript.

\section{Acknowledgments}

Similar analyses were performed on a partially common group of patients and presented as a poster on EASD Virtual Meeting, Barcelona 2019. This study was supported by the Statutory Work of the Medical University of Silesia.

\section{References}

[1] I. Marrocco, F. Altieri, and I. Peluso, "Measurement and clinical significance of biomarkers of oxidative stress in humans," Oxidative Medicine and Cellular Longevity, vol. 2017, Article ID 6501046, 32 pages, 2017.

[2] F. Giacco and M. Brownlee, "Oxidative stress and diabetic complications," Circulation Research, vol. 107, no. 9, pp. 1058-1070, 2010.

[3] C. Li, J. Zhang, M. Xue et al., "SGLT2 inhibition with empagliflozin attenuates myocardial oxidative stress and fibrosis in diabetic mice heart," Cardiovascular Diabetology, vol. 18, no. 1, p. 15, 2019.

[4] H. Osorio, I. Coronel, A. Arellano et al., "Sodium-glucose cotransporter inhibition prevents oxidative stress in the kidney of diabetic rats," Oxidative Medicine and Cellular Longevity, vol. 2012, Article ID 542042, 7 pages, 2012.

[5] S. J. Shin, S. Chung, S. J. Kim et al., "Effect of sodium-glucose co-transporter 2 inhibitor, dapagliflozin, on renal reninangiotensin system in an animal model of type 2 diabetes," PLoS One, vol. 11, no. 11, article e0165703, 2016.

[6] S. Steven, M. Oelze, A. Hanf et al., "The SGLT2 inhibitor empagliflozin improves the primary diabetic complications in ZDF rats," Redox Biology, vol. 13, pp. 370-385, 2017.

[7] S. Verma and J. J. V. McMurray, "SGLT2 inhibitors and mechanisms of cardiovascular benefit: a state-of-the-art review," Diabetologia, vol. 61, no. 10, pp. 2108-2117, 2018.

[8] V. Perkovic, D. de Zeeuw, K. W. Mahaffey et al., "Canagliflozin and renal outcomes in type 2 diabetes: results from the CANVAS program randomised clinical trials," The Lancet Diabetes and Endocrinology, vol. 6, no. 9, pp. 691-704, 2018.

[9] V. Perkovic, M. J. Jardine, B. Neal et al., "Canagliflozin and renal outcomes in type 2 diabetes and nephropathy," The New England Journal of Medicine, vol. 380, no. 24, pp. 22952306, 2019.

[10] B. Neal, V. Perkovic, K. W. Mahaffey et al., "Canagliflozin and cardiovascular and renal events in type 2 diabetes," The New England Journal of Medicine, vol. 377, no. 7, pp. 644-657, 2017.

[11] H. J. L. Heerspink, B. V. Stefánsson, R. Correa-Rotter et al., "Dapagliflozin in patients with chronic kidney disease," The New England Journal of Medicine, vol. 383, no. 15, pp. 14361446, 2020.

[12] B. Zinman, C. Wanner, J. M. Lachin et al., "Empagliflozin, cardiovascular outcomes, and mortality in type 2 diabetes," The New England Journal of Medicine, vol. 373, no. 22, pp. 2117-2128, 2015.

[13] S. D. Anker, J. Butler, G. Filippatos et al., "Effect of empagliflozin on cardiovascular and renal outcomes in patients with heart failure by baseline diabetes status: results from the EMPEROR-reduced trial," Circulation, vol. 143, no. 4, pp. 337-349, 2021.

[14] M. Brownlee, "The pathobiology of diabetic complications: a unifying mechanism," Diabetes, vol. 54, no. 6, pp. 1615$1625,2005$.

[15] G. Bresciani, I. B. da Cruz, and J. González-Gallego, "Manganese superoxide dismutase and oxidative stress modulation," Advances in Clinical Chemistry, vol. 68, pp. 87-130, 2015.

[16] P. Chelikani, I. Fita, and P. C. Loewen, "Diversity of structures and properties among catalases," Cellular and Molecular Life Sciences, vol. 61, no. 2, pp. 192-208, 2004. 
[17] L. Turell, R. Radi, and B. Alvarez, "The thiol pool in human plasma: the central contribution of albumin to redox processes," Free Radical Biology \& Medicine, vol. 65, pp. 244253, 2013.

[18] B. Kirschbaum, "Total urine antioxidant capacity," Clinica Chimica Acta, vol. 305, no. 1-2, pp. 167-173, 2001.

[19] I. Peluso and A. Raguzzini, "Salivary and urinary total antioxidant capacity as biomarkers of oxidative stress in humans," Pathology Research International, vol. 2016, Article ID 5480267, 14 pages, 2016.

[20] T. Cvetković, B. Mitić, G. Lazarević, P. Vlahović, S. Antić, and V. Stefanović, "Oxidative stress parameters as possible urine markers in patients with diabetic nephropathy," Journal of Diabetes and its Complications, vol. 23, no. 5, pp. 337-342, 2009.

[21] D. Il'yasova, P. Scarbrough, and I. Spasojevic, "Urinary biomarkers of oxidative status," Clinica Chimica Acta, vol. 413, no. 19-20, pp. 1446-1453, 2012.

[22] E. B. Kurutas, Y. Gumusalan, A. Cetinkaya, and E. Dogan, "Evaluation of method performance for oxidative stress biomarkers in urine and biological variations in urine of patients with type 2 diabetes mellitus and diabetic nephropathy," Biological Procedures Online, vol. 17, no. 1, pp. 3-7, 2015.

[23] O. Mutlu, D. Turgut Balik, and E. B. Kurutaş, "Kinetic analysis of the amino terminal end of active site loop of lactate deyhdrogenase from plasmodium vivax," Balkan Medical Journal, vol. 29, no. 4, pp. 364-369, 2012.

[24] X. Liu, W. Gan, Y. Zou et al., "Elevated levels of urinary markers of oxidative DNA and RNA damage in type 2 diabetes with complications," Oxidative Medicine and Cellular Longevity, vol. 2016, 7 pages, 2016.

[25] J. E. Davis, J. M. McDonald, and L. Jarett, "A highperformance liquid chromatography method for hemoglobin A1c," Diabetes, vol. 27, no. 2, pp. 102-107, 1978.

[26] J. R. Delanghe and M. M. Speeckaert, "Creatinine determination according to Jaffe - what does it stand for?," NDT Plus, vol. 4, no. 2, pp. 83-86, 2011.

[27] A. S. Levey, L. A. Stevens, C. H. Schmid et al., "A new equation to estimate glomerular filtration rate," Annals of Internal Medicine, vol. 150, no. 9, pp. 604-612, 2009.

[28] E. F. Beach and J. J. Turner, "An enzymatic method for glucose determination in body fluids," Clinical Chemistry, vol. 4, no. 6 , pp. 462-475, 1958.

[29] A. S. Hugget and D. A. Nixon, "Use of glucose oxidase, peroxidase, and O-dianisidine in determination of blood and urinary glucose," Lancet, vol. 270, no. 6991, pp. 368-370, 1957.

[30] R. Molinario, K. Pocino, P. D. Daloiso et al., "Urinary albumin detection: comparison of two different methods," Journal of Clinical Laboratory Analysis, vol. 30, no. 6, pp. 888-891, 2016.

[31] A. S. Levey, J. Coresh, K. Bolton et al., "K/DOQI clinical practice guidelines for chronic kidney disease: evaluation, classification, and stratification," American Journal of Kidney Diseases, vol. 39, 2 Suppl 1, pp. S1-266, 2002.

[32] Y. Oyanagui, "Reevaluation of assay methods and establishment of kit for superoxide dismutase activity," Analytical Biochemistry, vol. 142, no. 2, pp. 290-296, 1984.

[33] T. Adachi, H. Yamada, H. Hara, A. Futenma, and S. Kakumu, "Increase of urinary extracellular-superoxide dismutase level correlated with cyclic adenosine monophosphate," FEBS Letters, vol. 458, no. 3, pp. 370-374, 1999.
[34] O. Erel, "A novel automated direct measurement method for total antioxidant capacity using a new generation, more stable ABTS radical cation," Clinical Biochemistry, vol. 37, no. 4, pp. 277-285, 2004.

[35] J. F. Koster, P. Biemond, and A. J. Swaak, "Intracellular and extracellular sulphydryl levels in rheumatoid arthritis," Annals of the Rheumatic Diseases, vol. 45, no. 1, pp. 44-46, 1986.

[36] L. H. Johansson and L. A. Håkan Borg, "A spectrophotometric method for determination of catalase activity in small tissue samples," Analytical Biochemistry, vol. 174, no. 1, pp. 331336, 1988.

[37] G. Boudouris, I. I. Verginadis, Y. V. Simos et al., "Oxidative stress in patients treated with continuous ambulatory peritoneal dialysis (CAPD) and the significant role of vitamin C and E supplementation," International Urology and Nephrology, vol. 45, no. 4, pp. 1137-1144, 2013.

[38] A. F. Banne, A. Amiri, and R. W. Pero, "Reduced level of serum thiols in patients with a diagnosis of active disease," Journal of Anti-Aging Medicine, vol. 6, no. 4, pp. 327-334, 2003.

[39] S. Gupta, J. K. Gambhir, O. Kalra et al., "Association of biomarkers of inflammation and oxidative stress with the risk of chronic kidney disease in type 2 diabetes mellitus in north Indian population," Journal of Diabetes and its Complications, vol. 27, no. 6, pp. 548-552, 2013.

[40] A. M. Koning, W. C. Meijers, A. Pasch et al., "Serum free thiols in chronic heart failure," Pharmacological Research, vol. 111, pp. 452-458, 2016.

[41] M. M. Cortese-Krott, A. Koning, G. G. C. Kuhnle et al., "The reactive species interactome: evolutionary emergence, biological significance, and opportunities for redox metabolomics and personalized medicine," Antioxidants \& Redox Signaling, vol. 27, no. 10, pp. 684-712, 2017.

[42] H. Fujita, T. Sakamoto, K. Komatsu et al., "Reduction of circulating superoxide dismutase activity in type 2 diabetic patients with microalbuminuria and its modulation by telmisartan therapy," Hypertension Research, vol. 34, no. 12, pp. 1302 1308, 2011.

[43] R. K. Sundaram, A. Bhaskar, S. Vijayalingam, M. Viswanathan, R. Mohan, and K. R. Shanmugasundaram, "Antioxidant status and lipid peroxidation in type II diabetes mellitus with and without complications," Clinical Science (London, England), vol. 90, no. 4, pp. 255-260, 1996.

[44] M. E. Hartnett, R. D. Stratton, R. W. Browne, B. A. Rosner, R. J. Lanham, and D. Armstrong, "Serum markers of oxidative stress and severity of diabetic retinopathy," Diabetes Care, vol. 23, no. 2, pp. 234-240, 2000.

[45] H. M. Turk, A. Sevinc, C. Camci et al., "Plasma lipid peroxidation products and antioxidant enzyme activities in patients with type 2 diabetes mellitus," Acta Diabetologica, vol. 39, no. 3, pp. 117-122, 2002.

[46] M. Gul, E. Kurutas, P. Ciragil et al., "Urinary tract infection aggravates oxidative stress in diabetic patients," The Tohoku Journal of Experimental Medicine, vol. 206, no. 1, pp. 1-6, 2005.

[47] A. Mather and C. Pollock, "Glucose handling by the kidney," Kidney International. Supplement, vol. 79, pp. S1-S6, 2011.

[48] N. R. McSherry and S. F. Wen, "Segmental analysis of renal glucose transport in young female rats," The Journal of Physiology, vol. 356, no. 1, pp. 9-19, 1984.

[49] K. C. Linden, C. L. DeHaan, Y. Zhang et al., "Renal expression and localization of the facilitative glucose transporters GLUT1 
and GLUT12 in animal models of hypertension and diabetic nephropathy," American Journal of Physiology. Renal Physiology, vol. 290, no. 1, pp. F205-F213, 2006.

[50] C. L. Benn, P. Dua, R. Gurrell et al., "Physiology of hyperuricemia and urate-lowering treatments," Frontiers in Medicine, vol. 5, p. 160, 2018.

[51] T. Ramprasath, A. J. Freddy, G. Velmurugan et al., "Contextdependent regulation of nrf $2 /$ are axis on vascular cell function during hyperglycemic condition," Current Diabetes Reviews, vol. 16, no. 8, pp. 797-806, 2020.

[52] C. Tonelli, I. I. C. Chio, and D. A. Tuveson, "Transcriptional regulation by Nrf2," Antioxidants \& Redox Signaling, vol. 29, no. 17, pp. 1727-1745, 2018.

[53] Y. S. Oh and H. S. Jun, "Effects of glucagon-like peptide-1 on oxidative stress and nrf2 signaling," International Journal of Molecular Sciences, vol. 19, no. 1, p. 26, 2018.

[54] C. C. Sun, Y. N. Lai, W. H. Wang et al., "Metformin ameliorates gestational diabetes mellitus-induced endothelial dysfunction via downregulation of p65 and upregulation of Nrf2," Frontiers in Pharmacology, vol. 11, p. 575390, 2020.

[55] M. Maciejczyk, A. Zalewska, and J. R. Ładny, "Salivary antioxidant barrier, redox status, and oxidative damage to proteins and lipids in healthy children, adults, and the elderly," Oxidative Medicine and Cellular Longevity, vol. 2019, Article ID 4393460, 12 pages, 2019.

[56] A. Raghav, J. Ahmad, and K. Alam, "Preferential recognition of advanced glycation end products by serum antibodies and low-grade systemic inflammation in diabetes mellitus and its complications," International Journal of Biological Macromolecules, vol. 118, no. Part B, pp. 1884-1891, 2018.

[57] N. S. Sahajpal, R. K. Goel, A. Chaubey, R. Aurora, and S. K. Jain, "Pathological perturbations in diabetic retinopathy: hyperglycemia, ages, oxidative stress and inflammatory pathways," Current Protein \& Peptide Science, vol. 20, no. 1, pp. 92-110, 2018.

[58] R. Wada and S. Yagihashi, "Role of advanced glycation end products and their receptors in development of diabetic neuropathy," Annals of the New York Academy of Sciences, vol. 1043, no. 1, pp. 598-604, 2005.

[59] S. Del Turco and G. Basta, "An update on advanced glycation endproducts and atherosclerosis," BioFactors, vol. 38, no. 4, pp. 266-274, 2012.

[60] A. Nenna, F. Nappi, S. S. Avtaar Singh et al., "Pharmacologic approaches against advanced glycation end products (AGEs) in diabetic cardiovascular disease," Research in Cardiovascular Medicine, vol. 4, no. 2, article e26949, p. 5, 2015.

[61] G. Abbas, A. S. al-Harrasi, H. Hussain, J. Hussain, R. Rashid, and M. I. Choudhary, "Antiglycation therapy: discovery of promising antiglycation agents for the management of diabetic complications," Pharmaceutical Biology, vol. 54, no. 2, pp. 198-206, 2016.

[62] K. Adeshara and R. Tupe, "Antiglycation and cell protective actions of metformin and glipizide in erythrocytes and monocytes," Molecular Biology Reports, vol. 43, no. 3, pp. 195-205, 2016.

[63] K. M. Mil, M. E. Gryciuk, C. Pawlukianiec et al., "Pleiotropic Properties of Valsartan: Do They Result from the Antiglycooxidant Activity? Literature Review and In Vitro Study," Oxidative Medicine and Cellular Longevity, vol. 2021, Article ID 5575545, 20 pages, 2021.
[64] A. Ojima, T. Matsui, Y. Nishino, N. Nakamura, and S. Yamagishi, "Empagliflozin, an inhibitor of sodium-glucose cotransporter 2 exerts anti-inflammatory and antifibrotic effects on experimental diabetic nephropathy partly by suppressing ages-receptor axis," Hormone and Metabolic Research, vol. 47, no. 9, pp. 686-692, 2015.

[65] T. Eleftheriadis, G. Pissas, K. Tsogka, E. Nikolaou, V. Liakopoulos, and I. Stefanidis, "A unifying model of glucotoxicity in human renal proximal tubular epithelial cells and the effect of the SGLT2 inhibitor dapagliflozin," International Urology and Nephrology, vol. 52, no. 6, pp. 1179-1189, 2020.

[66] S. Maeda, T. Matsui, M. Takeuchi, and S. I. Yamagishi, "Sodium-glucose co-transporter 2-mediated oxidative stress augments advanced glycation end products-induced tubular cell apoptosis," Diabetes/Metabolism Research and Reviews, vol. 29, no. 5, pp. 406-412, 2013. 